\title{
Automation of the Complete Sample Management in a Biotech Laboratory
}

\author{
Martin Wojtczyk, Michael Marszalek and \\ Alois Knoll \\ Technische Universität München \\ Department of Informatics - \\ Robotics and Embedded Systems \\ Boltzmannstr. 3, \\ 85748 Garching b. München, Germany \\ wojtczyk@in.tum.de
}

\author{
Rüdiger Heidemann, Klaus Joeris, \\ Chun Zhang, Mark Burnett and \\ Tom Monica \\ Bayer HealthCare - Pharmaceuticals \\ Global Biological Development \\ 800 Dwight Way \\ Berkeley, CA, 94710, USA
}

\begin{abstract}
Both Robots and Personal Computers established new markets about $\mathbf{3 0}$ years ago and were enabling factors in Automation and Information Technology. However, while you can see Personal Computers in almost every home nowadays, the domain of Robots in general still is mostly restricted to industrial automation. Due to the physical impact of robots, a safe design is essential, which most robots still lack of and therefore prevent their application for personal use, although a slow change can be noticed by the introduction of dedicated robots for specific tasks, which can be classified as service robots.

Our approach to service robots was driven by the idea for supporting lab personnel in a biotechnology laboratory. That resulted in the combination of a manipulator with a mobile platform, extended with the necessary sensors to carry out a complete sample management process in a mammalian cell culture plant. After the initial development in Germany, the mobile manipulator was shipped to Bayer HealthCare in Berkeley, CA, USA, a global player in the sector of biopharmaceutical products, located in the San Francisco bay area. The platform was installed and successfully tested there in a pilot plant. This project demonstrates the successful combination of both key technologies: Information Technology and Robotics - and its application in a Life Science pilot plant.
\end{abstract}

Keywords: Robotics, Biotechnology, Informatics, Image processing, Video processing, Life sciences

\section{INTRODUCTION}

While facing a fast growing market for Biopharmaceuticals, Biotech companies need to comply with strongly regulated production processes, which often imply labour intensive tasks to control and to adjust growth parameters of recombinant cell lines. To overcome this enormous human effort, an autonomous mobile robot platform has been developed in a close cooperation between the University of Bielefeld, the Technische Universität München, Germany, and Bayer HealthCare in Berkeley, California, that is capable of automating the complete sample management in a biotechnological laboratory. After transferring the robot to the customer, it has been upgraded and customized to match local conditions and to serve the company's specific lab devices. Reliability and robustness have been demonstrated in 101 error free sampling cycles.

\section{BACKGROUND}

Sample management is an inevitable and time-consuming part during the development and production of biopharmaceuticals to keep track of growth parameters and to adjust these as it becomes necessary. Sampling and maintenance of cell culture processes are labor intensive and especially continuous perfusion operations require constant monitoring on a 24/7 basis. This was 
the driving force to develop a robot at the University of Bielefeld that is capable of carrying out this repetitive task [1, 2, 3]. However production setups in this kind of labs are very large and a robot would have to move long distances between distinct bioreactors and analysis devices. This challenge implies a mobile robot platform, as long as the given processes can not be optimised for short distances.

After initial construction and development at the University of Bielefeld, the project was transferred to the Technische Universität München, Germany, where development continued before the platform was finally shipped to the customer. Figure 1 shows the robot, as it arrived on site. Afterwards it was customised to match the local conditions of a cell culture pilot plant.

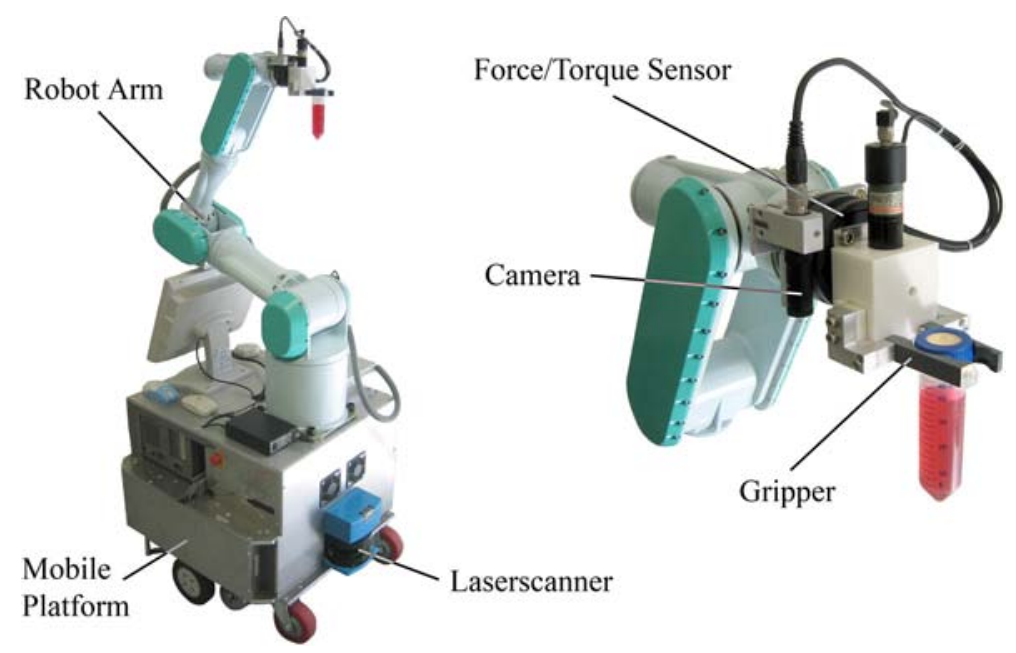

FIGURE 1: The mobile robot as it was shipped to Bayer HealthCare.

To support human lab personnel, the robot needs to be able to carry out the same tasks as a human and to serve the same or at least similar devices, without changing the entire lab equipment. Thus the robot was designed to be an autonomous, wheeled, mobile platform with two laser range finders for localisation, navigation and obstacle detection and powered by batteries. This makes the platform independent of any power supply for up to nine hours. Figure 2 shows the robot's view of the biotech lab through the laser range finders. Furthermore a kinematically redundant industrial robot arm with seven joints enables the robot to pick up, carry and place different sizes of sample vials even in close-packed areas. For precise interaction with the lab devices, the robot is also equipped with a camera to detect and localise objects like vials and analysis devices and a force-torque-sensor to prevent any damage by physical contact. All the sensors and effectors are controlled by an on-board computer which can receive user commands or give image, or other sensor feedback to an observing station or to invoke analysis processes at certain stations via wireless network [4, 5].

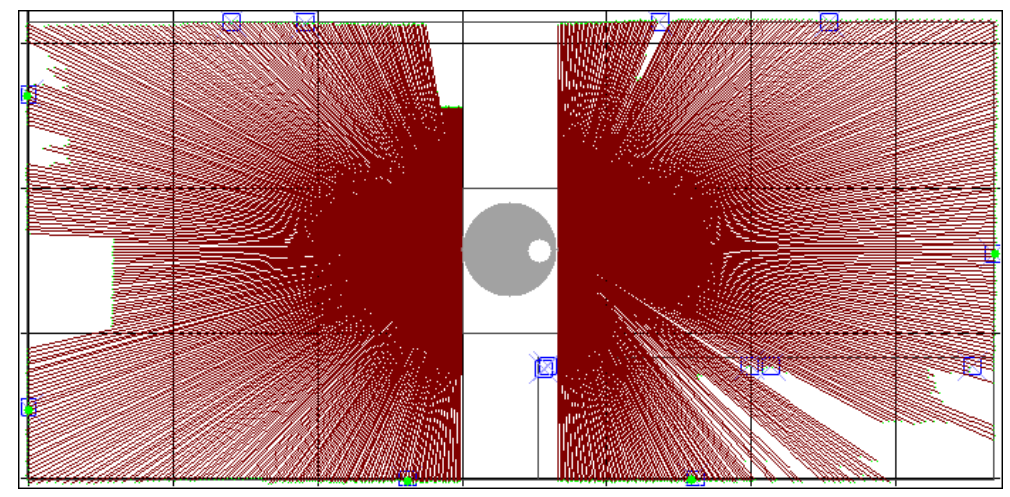

FIGURE 2: Localisation via the onboard Laser range finders. 
Precise device interaction is carried out as follows: to move to workstations, the robot positions itself using the laser range finders' feedback which is matched to the known map of the lab and some characteristic landmarks. Furthermore, to overcome precision issues of the platform, all relevant devices are tagged with color markers as shown in Figure 3 , which the robot approaches with the arm and its mounted tool, the camera constantly giving visual feedback for its actual position (see Figure 4). Finally, after reaching known positions, scripted movements can be triggered, which may be force controlled to prohibit damage due to physical interaction.
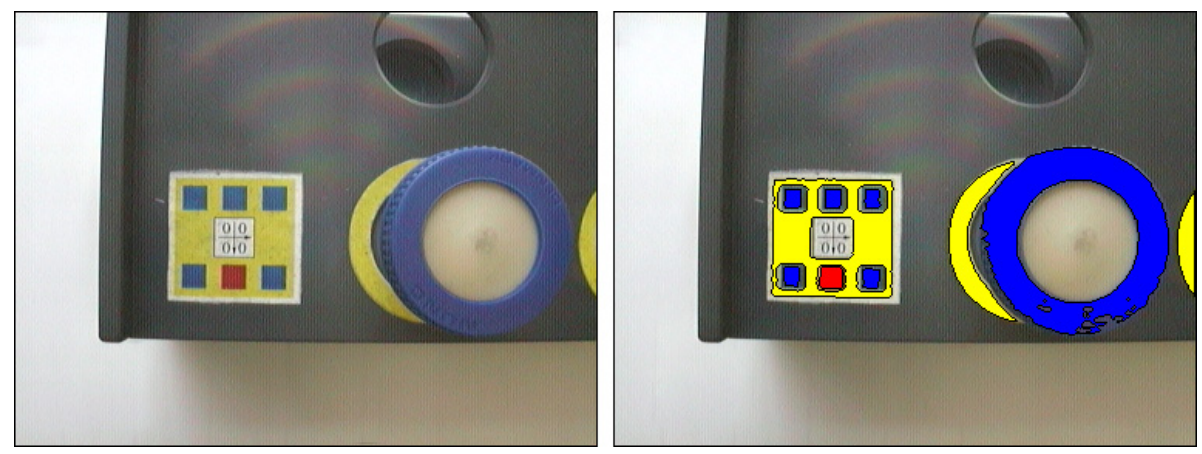

FIGURE 3: Image Processing using color features.

\section{CUSTOMISATION}

To serve the local requirements, the robot's tool has been replaced in a way that it can handle $10 \mathrm{ml}$ Vacutainer sample tubes as well as common $50 \mathrm{ml}$ Falcon 11 sample tubes and Cedex ${ }^{2}$ cups. The analog camera was replaced by a firewire camera to improve image quality, furthermore a Siemens/NERLITE ${ }^{3}$ ring illuminator was attached to the lens to improve the illumination in critical areas. Figure 5 shows the new tool of the robot arm.

The lab itself had a bioreactor and a Cedex Cell Counter. Depending on the cell sample a dilution step was necessary for the Cedex. Humans usually perform the dilution by hand utilising common manual pipettes. To enable the robot to carry out the dilution, a sample preparation station was constructed consisting of a Hamiltor ${ }^{4}$ PSD/2 electrical syringe connected to a Hamilton modular valve positioner serving the necessary liquids. The sampling in turn is performed by a dedicated pneumatic valve, which was constructed to take the sample vials. All lab devices were connected to controllers with serial or ethernet network interfaces, which enabled the robot to trigger specific actions, when required. Figure 6 shows the complete setup in the pilot plant, while figure 7 depicts the sample management process as an UML sequence diagram [6].

First, the robot invokes the sampling of the bioreactor. Once done, it takes the vial from the sampling valve. In the next step it places the vial at the sample preparation station and triggers the dilution process. In the meantime the robot picks up a new Cedex cup and places it at the dispenser needle of the preparation station, where $1 \mathrm{ml}$ of the sample is dispensed after complete preparation. Afterwards the robot places the Cedex cup at the Analyser and triggers the measurement process. Meanwhile the sampling vial is removed from the preparation station by the robot and placed in the waste basket, triggering an automated cleaning program of the preparation station. Furthermore the robot also removes the Cedex cup after it's analysation and places it in the biohazard waste basket. Finally the robot places a new $10 \mathrm{ml}$ sample vial in the cradle of the sampling valve, which leaves the setup ready for the next cycle [7].

\footnotetext{
http://www.bd.com/

2 http://www.innovatis.com/products_cedex

3 http://www.nerlite.com/

4 http://www.hamiltoncompany.com/
} 

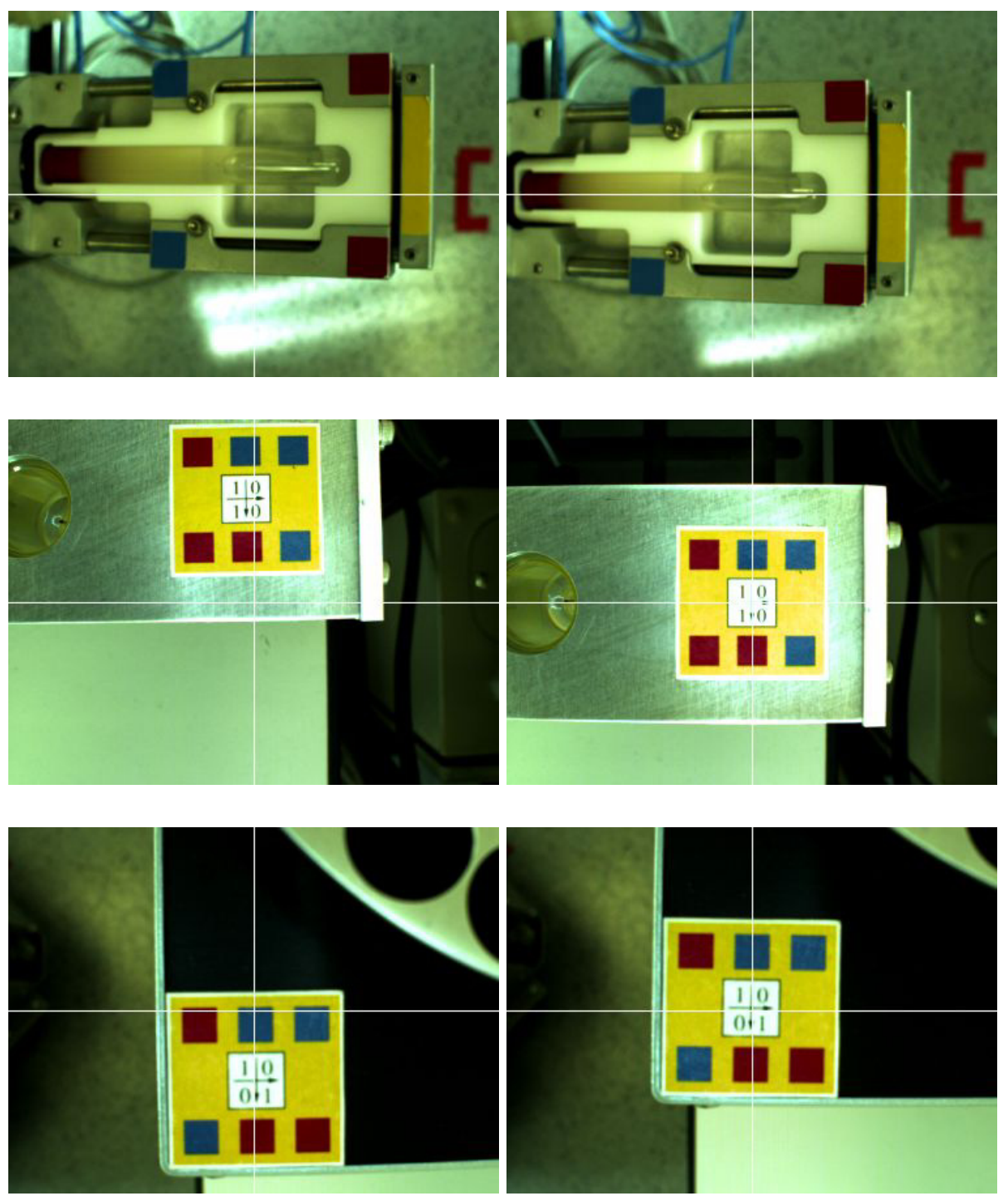

FIGURE 4: Three examples for approaching different lab devices - the sample valve (top), the sample preparation station (middle) and the Cedex cell counter (bottom) - and calibrating the robot arm's position through image processing.

\section{RESULTS}

To ensure repeatability, the implemented process was tested for its robustness. Therefore the robot was required to carry out 100 error free cycles. The sampling valve was driven pneumatically and equipped with steam connectors for sterilisation. It has been tested for approximately 2000 cycles without any mechanical issues, while the entire sample management process was carried out successfully for 101 times. Out of that number, 75 cycles were executed with real cell samples to measure deviations and the physical stress caused by the automated sampling and sample preparation. The figures 8 and 9 show eight viability and viable cell density (VCD) measurements per sample comparing the robot's results with two manual results side by side - one manual result with the sample taken out of the original $50 \mathrm{ml}$ sample vial and one out of the Vacutainer vial, which the robot had just used. While the graphs show good overall comparability, especially with the cells from cell line 2 , which seem to be less sensitive, the robot's results in general appear to be slightly lower. The reason for this probably resides in the higher number of syringe strokes at the sample preparation station utilised by the robot. 


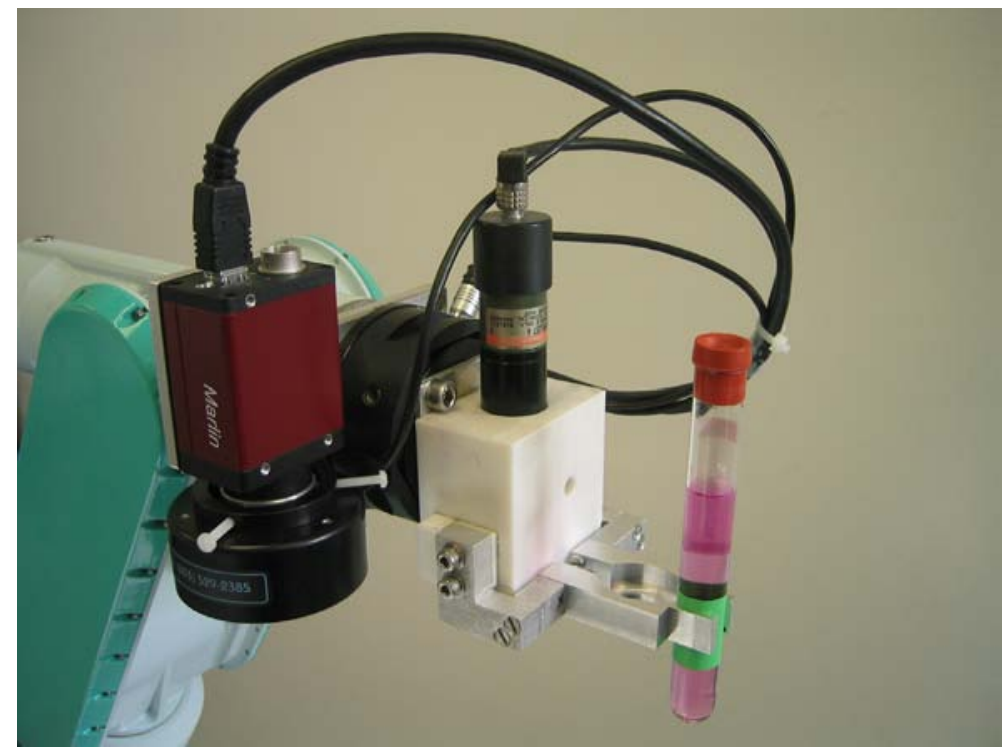

FIGURE 5: The robot's new tool with a firewire camera and a ring illuminator surrounding the lens. The adjusted gripper carries one of the new $10 \mathrm{ml}$ sample vials.

\section{WORK IN PROGRESS}

While the previously shown results of the experiments and tests, which were carried out, look very promising that the presented system is in fact able to carry out the required sample management process, the vision is to provide a service robot, which is able to work side by side with human personnel without programming the knowledge about the environment. Right now, the presented system has a static map and approximately knows where devices are located. It would just pause its movements if it encounters a dynamic obstacle like a human operator. Although the lab devices are slightly augmented with markers for easier recognition and precise calibration, the system is versatile enough, to utilise the same vials and devices as humans which is an important step towards the desired service robot.

However one may argue, that a service robot should be more context aware and that it should adaptively adjust its knowledge base to its environment. For the first steps towards these improvements we thought of an easier scenario than the sample management process. Utilising the mobile platform as a surveillance robot allows us to apply recently developed methods to the system. It should be able to explore the accessible lab space by itself thus automatically creates a map and utilises it for localisation. Furthermore marker-less lab devices and utilities should be identified and localised on-the-fly by inspecting the pictures and picture sequences acquired with the camera. Hence the robot could perform automated lab walkthroughs, inspecting bioreactors, pumps and connected hoses for example and trigger an alarm if a failure is detected. Once notified, a user could just log on to the robot and use its camera for remote inspection via the network, even via the internet. The surveillance scenario was also chosen for the implicitly reduced device interaction.

\subsection{Localisation and Mapping}

With regard to the mentioned goals, current work in progress also addresses the combined problem of simultaneous localisation and mapping (SLAM) where particle filter based, probabilistic approaches have shown very promising results. Based on DP-SLAM, an improved software package has been developed which was utilised successfully on a Robotin ${ }^{5}$ mobile robot with a SICK LMS 200 ${ }^{6}$ and a Laptop attached to it [8, 9].

\footnotetext{
5 http://www.festo-didactic.com/int-en/learning-systems/new-robotino/ ' http://www.sick.com/home/factory/catalogues/auto/lmsindoor/en.html
} 


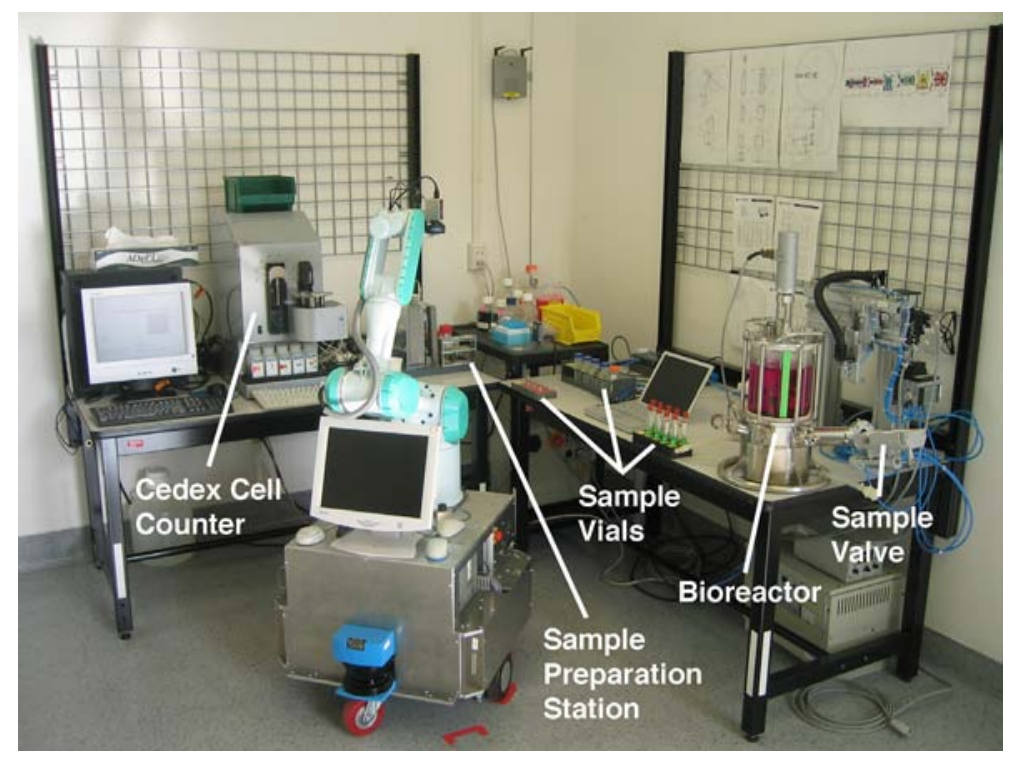

FIGURE 6: The robot lab for the cell counting sample management process with all necessary devices.

The algorithm needs only an accurate laser rangefinder and odometry measurements for localization and building a map. It uses a particle filter to maintain a joint probability distribution over maps and robot positions, as well as some efficient data structures which allow a fast mapping. A particle describes a posssible position of the robot in its environment. Another important fact is that this algorithm needs no information about the environment and produces extremely good maps with a minimal misalignment error. In comparision to other approaches, DP-SLAM does not need predetermined landmarks and is accurate enough to close loops without any special off-line techniques [8]. The data association problem was also eliminated through the abandonment of landmarks. Moreover it is not necessary to predetermine the environment.

The core data structures are an ancestry tree and an occupancy grid which enable an efficient and fast mapping process. The environment is subdiveded into a simple array or a grid of rectangular cells. The resolution of the environment mapping depends of the size of the cells. Additionally, a probabilistic measure of occupancy is associated with each cell. This measure marks the cell as occupied or unoccupied. A grid square consists of an ancestry tree which stores all particles which have updated this grid square and illustrates an intern storage of a map.

While most particle filter based SLAM methods require substantial resources in terms of memory and computing power, our solution has been andapted for running in live mode on currently available off the shelf PCs or Laptops and for easily adjusting the necessary resource parameters to provide an appropriate solution for different mobile platforms. A new version of the developed software package will be tested in combination with other data structures in order to advance the performance of the mapping process in terms of computing time. Figures 10 and 11 show sample maps which were acquired in live mode with the mentioned Robotino setup. This software package is currently being installed on the lab robot for its first industrial application.

\subsection{Visual Tracking}

The second aspect which is currently being improved is the vision system, which relied on coloured markers for easier identification and localisation of lab devices. Our computer vision group has been developing "a unifying software architecture for model-based visual tracking", which has been used successfully in a multi camera setup for tracking people in a real world TV Studio in Cologne, Germany [10, 11, 12]. For the lab robot we are utilising another methodology of the library, which allows, given a CAD model of a known object, detecting and tracking it in realtime with six degrees of freedom. 


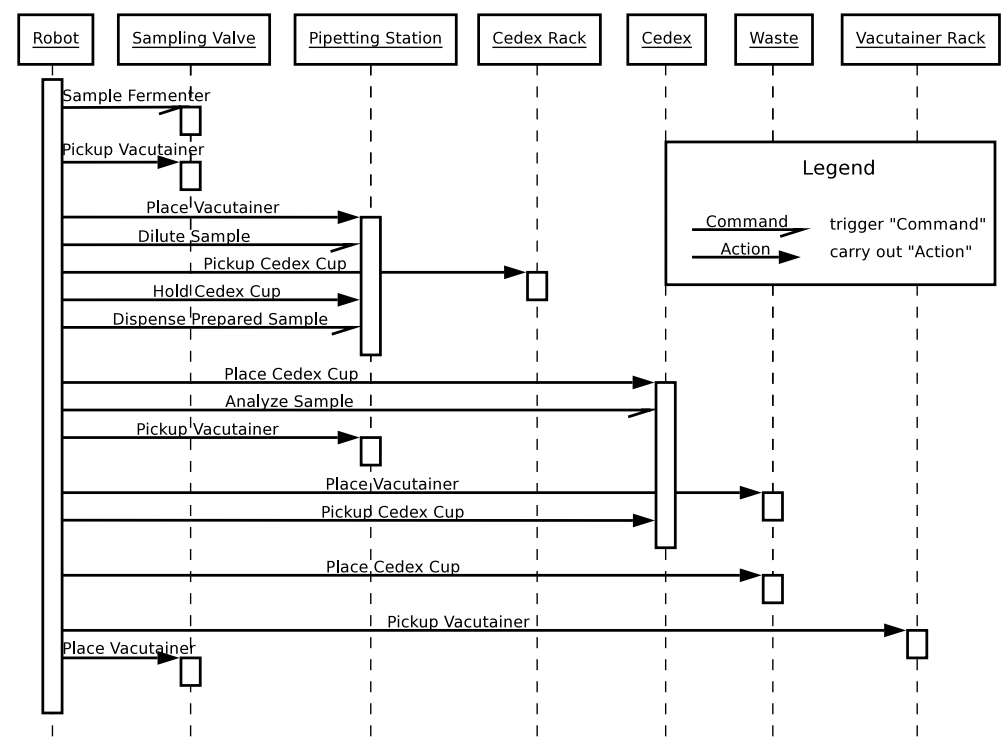

FIGURE 7: The implemented sample management process as an UML sequence diagram. The boxes on the top represent the devices, which the robot needs to operate. The two different kind of arrows symbolise commands, which are triggered by the robot and actions which the robot carries out itself.

\section{FUTURE WORK}

While the improvements from section 5 will dramatically enhance the context awareness and the usability of the robot, it is apparent that the surveillance scenario implies less device interaction than the sample management process in a controlled environment. The surveillance scenario was chosen as a next step because many problems in robotics are still not generally resolved, yet it allows applying recent research results in localisation, mapping and computer vision. Furthermore, to meet the requirements of a safe service robot, current research work addresses on-line 3D reconstruction to adapt a robot's geometrical world model to its environment which in turn can be used for collision-free path-planning for the mobile manipulator, but even given a perfect world model, an on-line and collision-free path-planning for manipulators with many degrees of freedom is a challenging task. Among others, these are some requirements which enable robots to safely interact with the physical world without prior knowledge.

\section{CONCLUSION}

This report describes an approach to combine both fields: Robotics and Computer Science for an application in Life Sciences; the environmental challenges and accomplished steps for providing a platform, which is available on a $24 / 7$ basis for constant, robust and reproducible monitoring of continuous perfusion processes in cell culture development. The results show very comparable results as if the process was carried out manually.

Subsequent improvements are addressed to enable the robot operate in a changeable laboratory shared with human lab personnel and to provide a demonstrator for the transition from industrial automation towards service robotics. This is one successful example and a real world application combining key technologies in Life Science and Computer Science utilising Robotics as the link between Information Technology and the physical world.

\section{REFERENCES}

[1] Lütkemeyer, D. et al., Biotechnology Progress 16 (2000) 822.

[2] Scherer, T. et al., Emerging Technologies and Factory Automation, 2003. Proceedings. ETFA '03. IEEE Conference 2 (2003) 383. 
[3] Knoll, A., Scherer, T., Poggendorf, I., Lütkemeyer, D., and Lehmann, J., Biotechnology Progress 20 (2004) 1825.

[4] Scherer, T., A Mobile Service Robot for Automisation of Sample Taking and Sample Management in a Biotechnological Pilot Laboratory, Dissertation, University of Bielefeld, 2004.

[5] Poggendorf, I., Einsatz eines Serviceroboters zur Automatisierung der Probenentnahme und des Probenmangements während Kultivierungen tierischer Zellen in einer Technikumsumgebung, Dissertation, Universität Bielefeld, 2004, http://nbn-resolving.de/urn/resolver. pl?urn=urn:nbn:de: hbz:361-5651.

[6] Ambler, S. W., The Object Primer: Agile Model-Driven Development with UML 2.0, Cambridge University Press, 2004.

[7] Wojtczyk, M. et al., The Use of a Mobile Robot for Complete Sample Management in a Cell Culture Pilot Plant, in Cell Technology for Cell Products, Proceedings of the 19th ESACT Meeting, Harrogate, UK, June 5-8, 2005, edited by Smith, R., pages 543-547, Springer Netherlands, 2007.

[8] Eliazar, A. and Parr, R., DP-SLAM: Fast, robust simultaneous localization and mapping without predetermined landmarks, 2003.

[9] Marszalek, M. L., Simultaneous Localization and Mapping Methods in Combination with Machine Learning Methods, Master's thesis, Technische Universität München, München, 2008.

[10] Panin, G. et al., A unifying software architecture for model-based visual tracking, in Image Processing: Machine Vision Applications. Edited by Niel, Kurt S.; Fofi, David. Proceedings of the SPIE, Volume 6813, pp. 681303-681303-14 (2008)., volume 6813 of Presented at the Society of Photo-Optical Instrumentation Engineers (SPIE) Conference, 2008.

[11] Nair, S. et al., A Multi-Camera Person Tracking System for Robotic Applications in Virtual Reality TV Studio, in 2008 IEEE/RSJ International Conference on Intelligent Robots and Systems, Acropolis Convention Center, Nice, France, 2008, Accepted for publication.

[12] Panin, G., Ladikos, A., and Knoll, A., An efficient and robust real-time contour tracking system, in ICVS '06: Proceedings of the Fourth IEEE International Conference on Computer Vision Systems, page 44, Washington, DC, USA, 2006, IEEE Computer Society. 


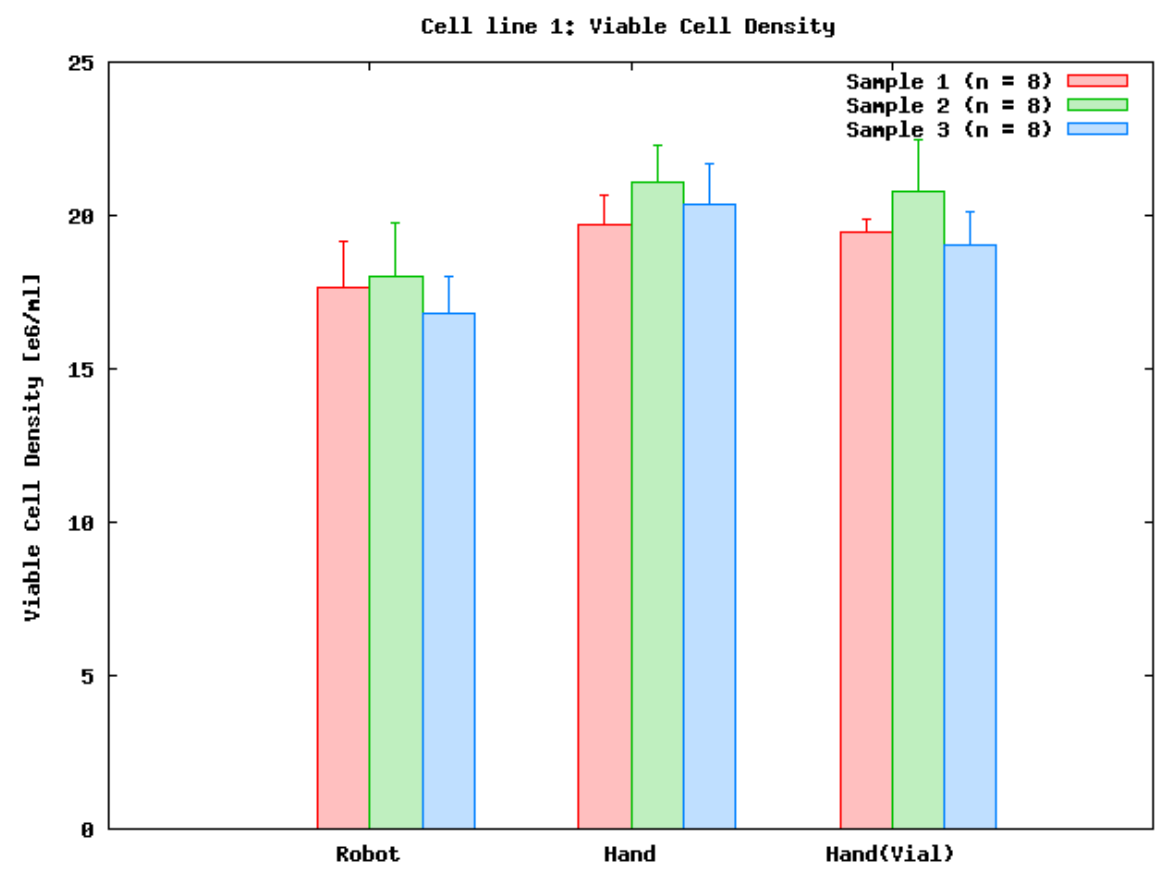

Cell line 1: Viability

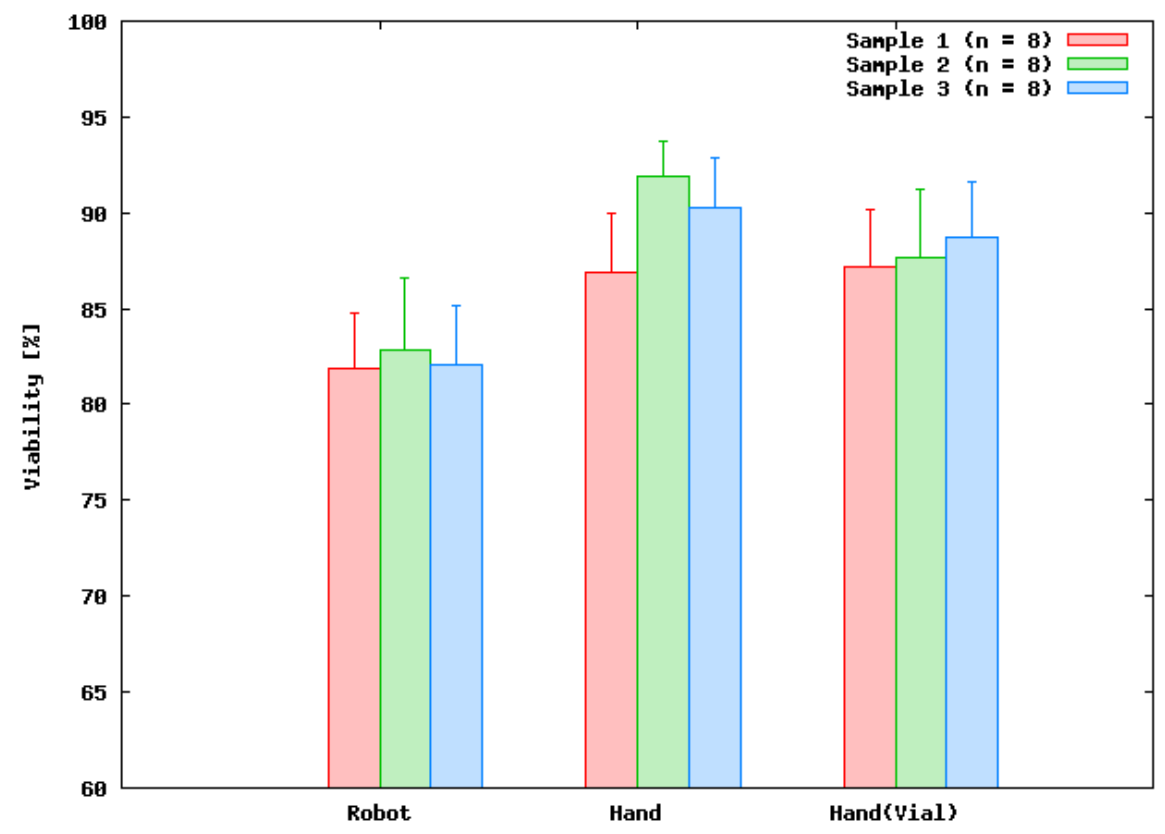

FIGURE 8: Comparison of the Viable Cell Density and Viability from three different samples of Cell line 1. Each bar represents the average and the standard deviation of eight measurements with the same cell sample. Furthermore the measurements are split into three sections: measured by the robot (Robot), measured manually out of the original cell source at almost the same time (Hand) and measured manually out of the $10 \mathrm{ml}$ vial, that the robot had just used (Hand(Vial)). The results show slightly lower viable cell density and viability values when measured by the robot. This may be a result of the increased stress and higher number of syringe strokes at the sample preparation station compared to manual pipetting, which may have an impact on this cell line in particular. 

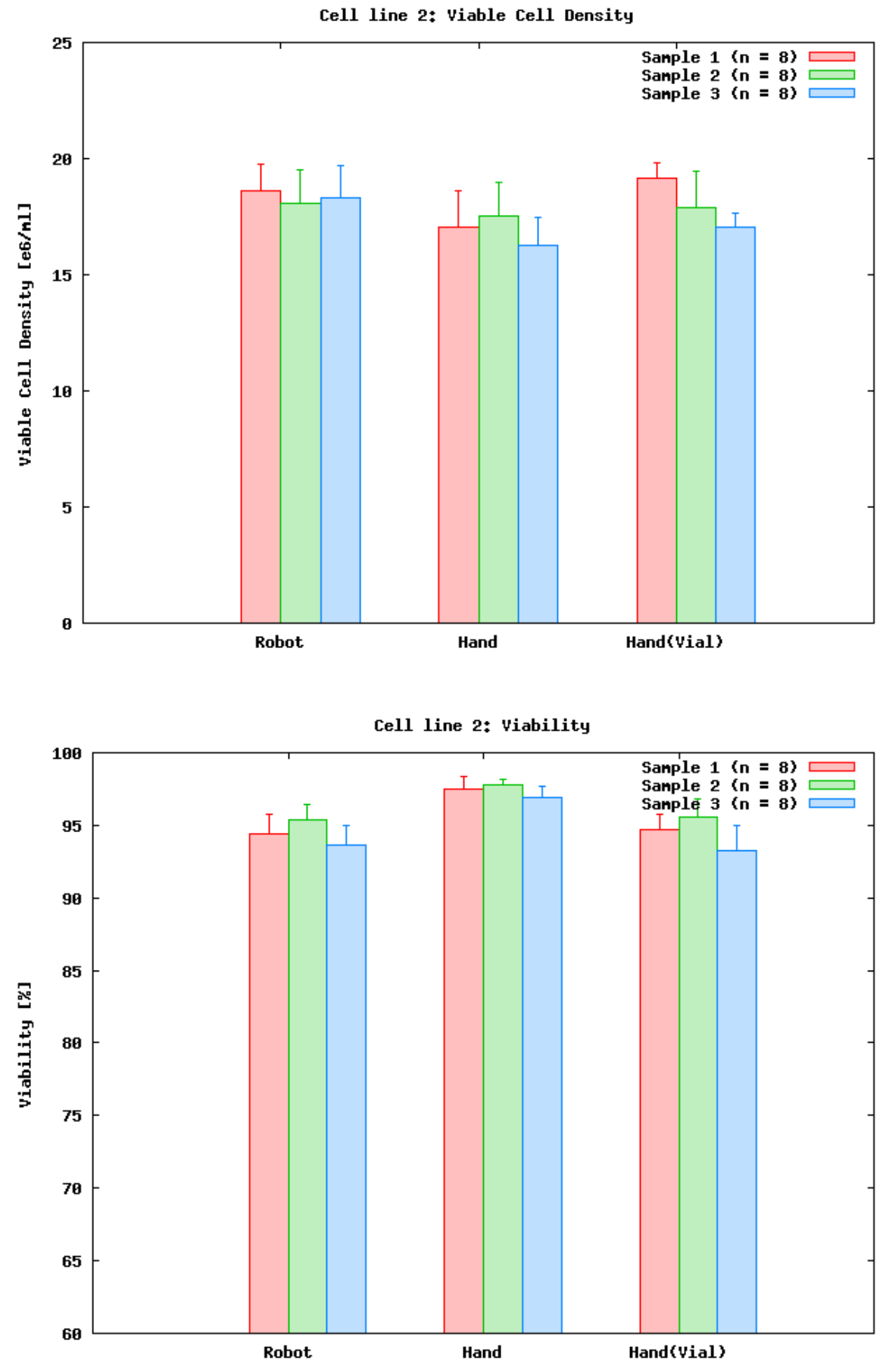

FIGURE 9: Comparison of the Viable Cell Density and Viability from three different samples of Cell line 2. Each bar represents the average and the standard deviation of eight measurements with the same cell sample. Furthermore the measurements are split into three sections: measured by the robot (Robot), measured manually out of the original cell source at almost the same time (Hand) and measured manually out of the $10 \mathrm{ml}$ vial, that the robot had just used (Hand(Vial)). The results show no significant difference between the robot's measurements and the manual measurements. 


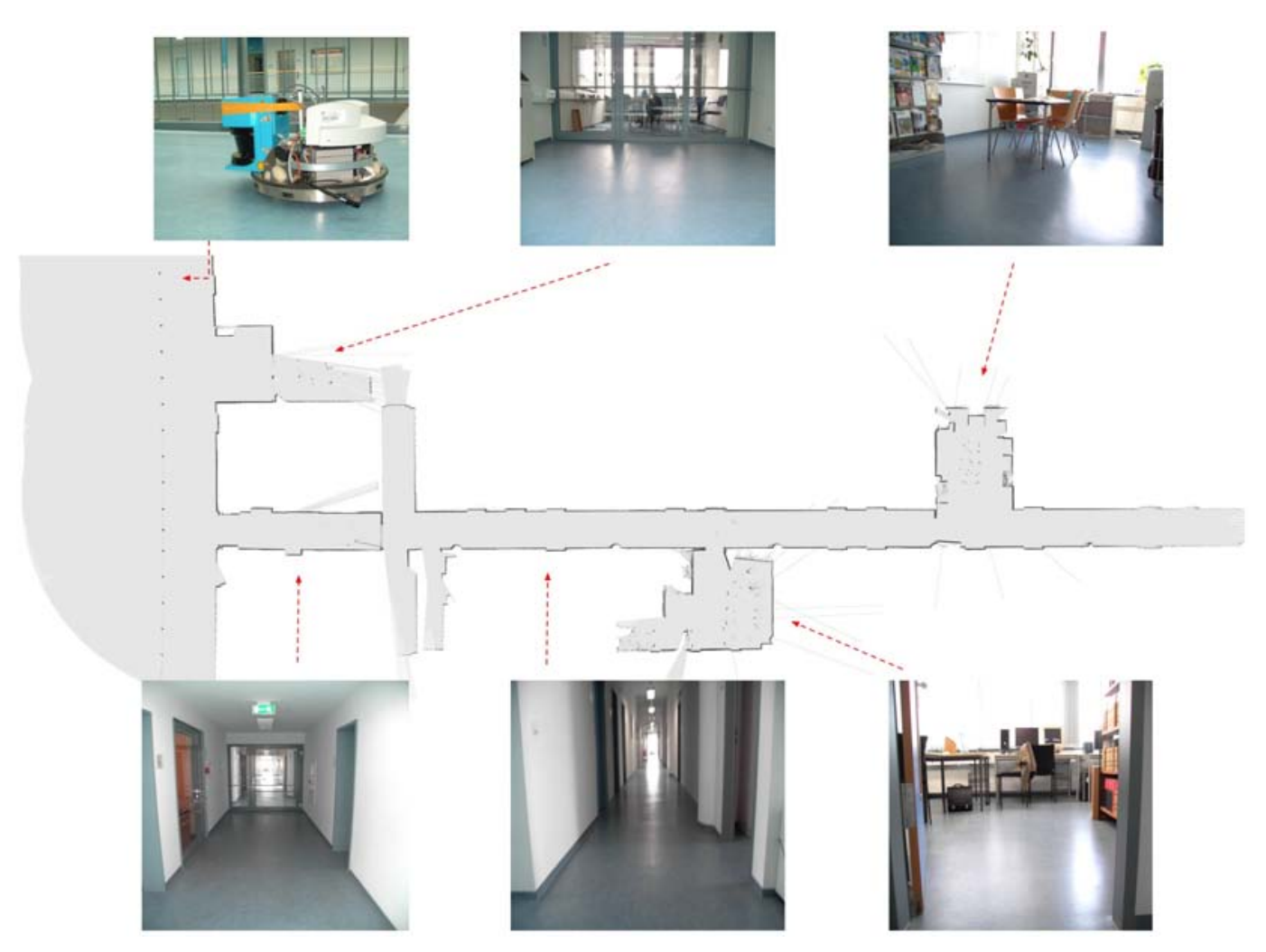

FIGURE 10: The automatically generated map of the Department of Informatics: Robotics \& Embedded Systems at the Technische Universität München.
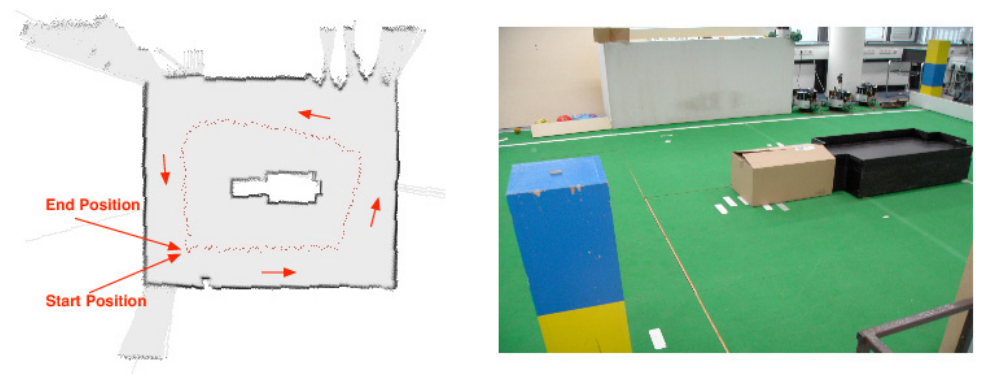

FIGURE 11: The map of the robot soccer field at the chair with an obstacle in the middle demonstrating the appropriate closure of loops. The red dots symbolise the robot's localisations. 